\title{
Spontaneous Coronary Artery Dissection: Efficient Clinical Outcome through Percutaneous Coronary Intervention
}

\author{
Hong-Cheng $\mathrm{Mai}^{1}$, Dan Lu${ }^{1}$, Ai-Dong Zhang ${ }^{2}$ and Tao Zhang ${ }^{* 2}$ \\ ${ }^{1}$ Department of Internal Neurology, Guangzhou Overseas Chinese Hospital, The First Affiliated Hospital of JiNan \\ University, Guangdong, China \\ ${ }^{2}$ Department of Internal Cardiology, Guangzhou Overseas Chinese Hospital, The First Affiliated Hospital of JiNan \\ University, Guangdong, China
}

${ }^{*}$ Corresponding author: Tao Zhang, Department of Internal Cardiology, Guangzhou Overseas Chinese Hospital, The First Affiliated Hospital of JiNan University, Guangzhou, Guangdong, China 510632, E-mail: heartdiease@126.com

Citation: Hong-Cheng Mai, Dan Lu, Ai-Dong Zhang, Tao Zhang (2016) Spontaneous Coronary Artery Dissection: Efficient Clinical Outcome through Percutaneous Coronary Intervention. J Case Rep Stud 4(1): 103. doi: $10.15744 / 2348-9820.4 .103$

Received Date: October 28, 2015 Accepted Date: February 11, 2016 Published Date: February 15, 2016

\begin{abstract}
Spontaneous coronary artery dissection (SCAD) is a rare myocardial ischemic disease that threatens patients' life. Various risk factors are associated with SCAD, such as smoking, severe hypertension and psychological reasons. Considering the formation of dissection, SCAD can be divided into intimal tear type or intraluminal hemorrhage type. The clinical presentation of SCAD depends on the extent and severity of progression of dissection and may present as unstable angina, acute myocardial infarction, ventricular arrhythmias or asymptom. Treatment of SCAD usually includes percutaneous coronary intervention (PCI), bypass graft surgery or medical therapy depending on the range of dissection. Herein, we report a 70 years-old male diagnosed with dissection in the left anterior descending artery by coronary arteriography. Despite the patient's exposure to the ventricular arrhythmias, he had a good prognosis and survived at 2 years of following up. We found PCI treatment is suitable for the patient who had SCAD.
\end{abstract}

Keywords: Spontaneous coronary artery dissection; Percutaneous coronary intervention; Left anterior descending artery

\section{Case Presentation}

A 70 years-old male with a history of diabetes mellitus and hypertension, presented an acute squeezing chest tight of 5 hours duration in precordium after emotional stress. He was admitted to emergency department with blood pressure 171/100 $\mathrm{mmHg}$. Electrocardiogram (ECG) showed frequent ventricular premature contractions and complete left bundle branch block which did not happen before. Laboratory examination showed elevated cardiac Troponin I ( $0.83 \mu \mathrm{g} / \mathrm{L}$ reference range, 0 to $0.15 \mu \mathrm{g}$ per liter) Creatine Kinase (268 U/L reference range, 30 to $170 \mathrm{IU}$ per liter) and Creatine Kinase isoenzyme MB (31 U/L reference range, 0 to $25 \mathrm{IU}$ per liter). Pharmacological management were included nitrate (Isosorbide mononitrate and glonoin) before primary percutaneous coronary intervention (PCI), His coronary angiogram showed a significant about $15 \mathrm{~mm}$ lesion, dissection at the proximal left anterior descending artery(LAD) (Figure1) and diffuse severe stenosis in middle part of right coronary artery (RCA). There were no signs of dissection in the left circumflex coronary (LCx). Our strategy was combining pharmacological management and PCI which mean implanting a stent in the dissection of LAD. The procedure of cardiac catheterization was supported by intraaortic balloon pump (IABP) and temporary pacemaker (TPM), considering the patient with frequent ventricular premature and heart failure [ejection fraction $28 \%$ detected by transthoracic echocardiogram (TTE)]. Firstly a $40 \mathrm{ml}$ IABP was implanted in aorta with adjusted counterpulsation pressure 110 to $130 \mathrm{mmHg}$. Then TPM was transported in the right ventricular apex with $60 \mathrm{t} / \mathrm{min}$ setted. A Stent $(4.0 \times 24 \mathrm{~mm})$ was successfully implanted after balloon dilated from the LAD near end. Repeat angiography revealed that the LAD dissection noted earlier was resolved and far-end flowing blood improved after 30min observation. The patient continued the therapy by treating dual anti-platelet (oral aspirin 100mg and clopidogrel 75mg), blood pressure controlled (oral perindopri $2 \mathrm{mg})$, and blood sugar controlled. After 1 week observation, we decided to implant two Stents $(3.5 \times 30 \mathrm{~mm} 、 4.0 \times 18 \mathrm{~mm})$ in the RCA to improve the far-end flowing (Figure 3). Meanwhile we did a angiography which also showed LAD dissection disappeared and no stenosis caused again (Figure 2). The patient did not complain discomfort through the procedure. He was maintained dual anti-platelet blood pressure and blood sugar controlled after revascularization. TTE showed a nearly normal ejection fraction (48\%) after 2 years of following up. 

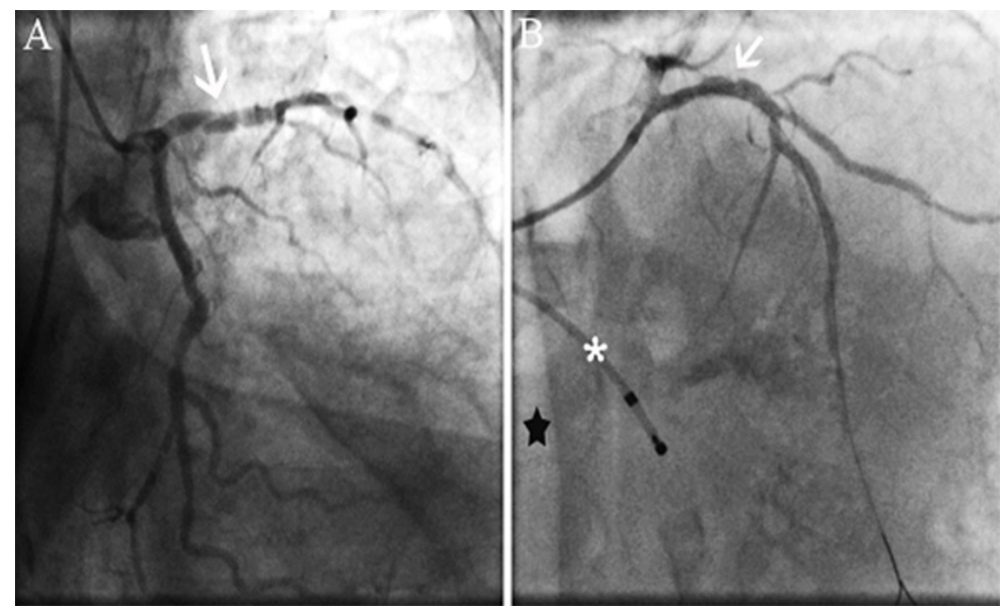

Figure 1: Coronary angiography signed: A) Cranial projection showing double-lumen (arrow) with contrast dye stained in the proximal LAD and radiolucent intimal flap lines; B) Caudal projection showing double-lumen (arrow) with contrast dye stained in the proximal LAD. Intra-aortic balloon pump; IABP (pentagram) and temporary pacemaker; TPM (asterisk) SCAD, spontaneous coronary artery dissection; LAD, left descending artery
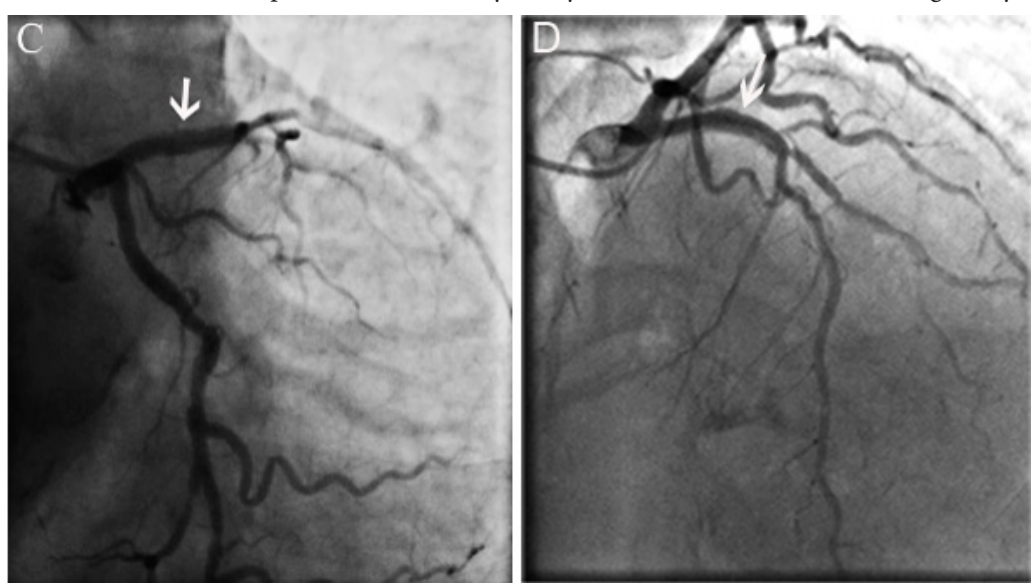

Figure 2: Follow-up coronary angiography after one week: cranial projection $\mathbf{C}$ ) and caudal projection; D) revealed almost complete healing of the dissection in LAD and no residual stenosis after stent implanted
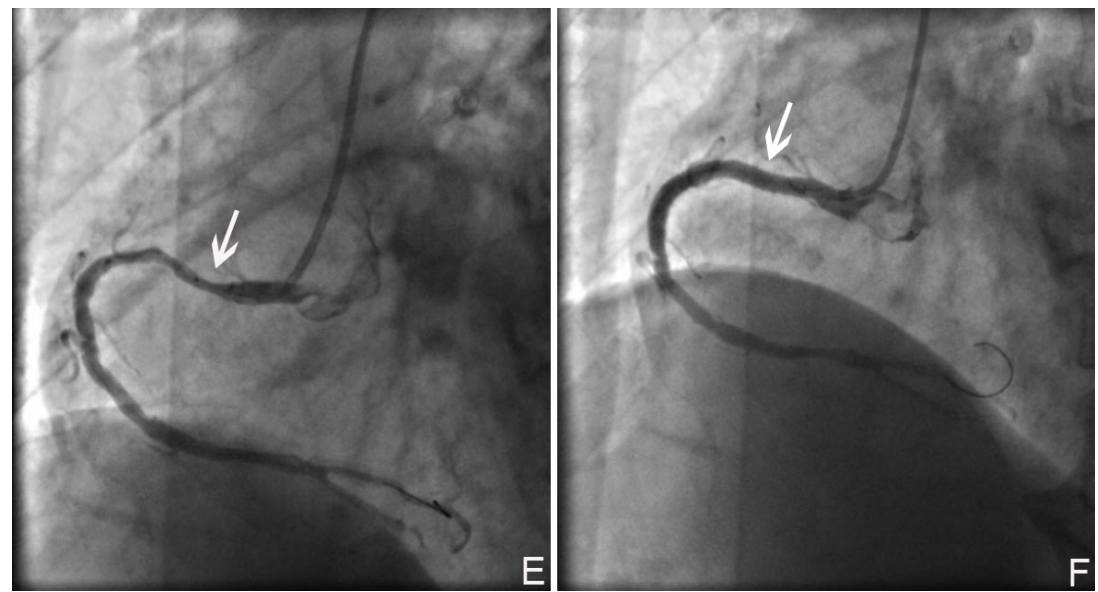

Figure 3: Right coronary artery (RCA) coronary angiography: stenosis in RCA E) Image of RCA F) after resolution

\section{Discussion}

In this case, our patient newly diagnosed with SCAD presented with two rare conditions; SCAD likely responsible for his ventricular arrhythmias and resulting in heart failure.

SCAD is defined as a rare but potentially fatal condition that coronary arterial wall is separated by hemorrhage. It often causes sudden coronary syndrome, ventricular arrhythmias, or acute myocardial infraction [1].Although SCAD was a concealed disease, several imaging approaches can diagnosis and confirm efficiently. For example, selective coronary angiography which showed double lumen with contrast dye stained the LAD by cranial projection or caudal projection is easy to identify (Figure 1). Also SCAD can be further confirmed by radiolucent intimal flap lines (Figure 1) [2]. Both intravenous ultrasound (IVUS) and optic coherence tomography (OCT) which are excellent to assess the visible arterial wall structure and composition can use as supplement angiographic $[3,4]$. The applications are limited for they would increase the economic burden on patients. 
Some studies and case reports have shown that several conditions have been associated with SCAD, such as postpartum, connective tissue diseases, and atherosclerosis [5]. According to associated predisposing condition, SCAD is divided into intimal tear type (related with atherosclerosis) or intraluminal hemorrhage type (related with non-atherosclerosis) [2]. Approximately 70\% of dissection happened in a single coronary artery and very rare can be found in multi vessel. No matter LAD, LCX or RCA also have sensitivity to SCAD. Normally RCA is easier to be found in males 'SCAD [6]. In the prensent case, our patient presented significant intimal tear type dissection in LAD. Compared to other normal cases, our patient rarely has a severe atherosclerotic coronary stenosis in RCA (Figure 3) at the same time which made the treatment become more difficult.

The optimal treatment strategy for this case remains a big challenge. The decision should be made by the clinical status of the patient which had ventricular arrhythmias and the range of dissection that is intimal tear type. In the retrospective review, conservative treatment is preferred for patients who are stable and without ongoing pain. Patients should undergo feasible PCI for the most important lesions which affected left main or proximal segments of LAD [7]. Emergency CABG should be considered if PCI failed or dissection is complicated. As our patient was in an unstable condition and the dissection located in proximal segments of LAD, we decided to stent only the site of lesion in LAD at that time. After stent implanted in LAD one week, we considered solving problem of the stenosis in RCA(Fig.3). During the procedure of cardiac catheterization, an angiography showed the flow in the remainder of LAD was normal that suggested PCI is associated with favorable outcome for this patient.

\section{Conclusion}

A case of SCAD triggered by an emotional stress factor in a 70 years-old male with ventricular arrhythmias and heart failure is described. The initial CAG revealed sign of double lumen with contrast dye filling in the LAD, where the LAD dissection and RCA stenosis are revascularized after stent implanted. This case suggested that PCI treatment strategy of SCAD in patient with RCA stenosis showed to be safe and effective.

\section{References}

1. Saw J (2013) Spontaneous coronary artery dissection. Can J Cardiol 29: 1027-33.

2. Saw J (2014) Coronary angiogram classification of spontaneous coronary artery dissection. Catheter Cardiovasc Interv 84: 1115-22.

3. Maehara A, Mintz GS, Castagna MT, Pichard AD, Satler LF, et al. (2002) Intravascular ultrasound assessment of spontaneous coronary artery dissection. Am J Cardiol 89: 466-8.

4. Alfonso F, Paulo M, Gonzalo N, Dutary J, Jimenez-Quevedo P, et al. (2012) Diagnosis of spontaneous coronary artery dissection by optical coherence tomography. J Am Coll Cardiol 59: 1073-9.

5. Tweet MS, Hayes SN, Pitta SR, Simari RD, Lerman A, et al. (2012) Clinical features, management, and prognosis of spontaneous coronary artery dissection. Circulation 126: 579-88.

6. Kansara P, Graham S (2011) Spontaneous coronary artery dissection: case series with extended follow up. J Invasive Cardiol 23: $76-80$.

7. Yip A, Saw J (2015) Spontaneous coronary artery dissection-A review. Cardiovasc Diagn Ther 5: 37-48.

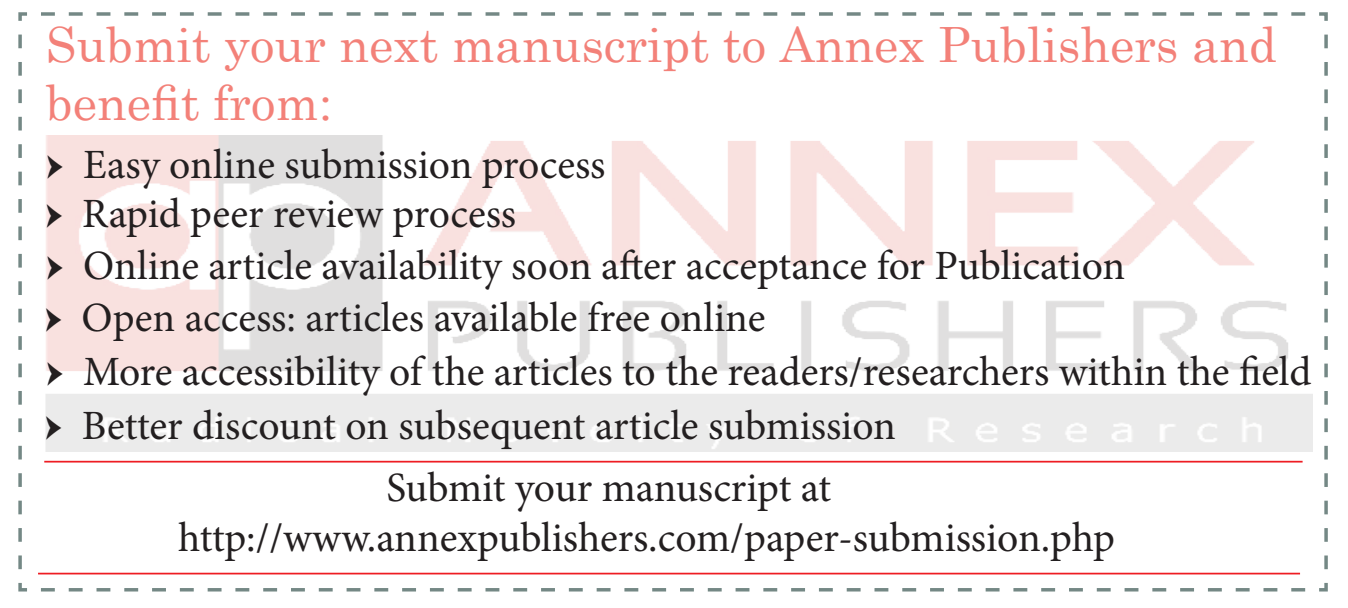

